\title{
Magnetospheric reconnection driven by solar wind pressure fronts
}

\author{
A. Boudouridis ${ }^{1}$, E. Zesta ${ }^{1}$, L. R. Lyons ${ }^{1}$, P. C. Anderson ${ }^{2}$, and D. Lummerzheim ${ }^{3}$ \\ ${ }^{1}$ Department of Atmospheric Sciences, University of California, Los Angeles, USA \\ ${ }^{2}$ Space Science Applications Laboratory, The Aerospace Corporation, Los Angeles, USA \\ ${ }^{3}$ Geophysical Institute, University of Alaska, Fairbanks, USA
}

Received: 16 April 2003 - Revised: 31 July 2003 - Accepted: 14 November 2003 - Published: 2 April 2004

\begin{abstract}
Recent work has shown that solar wind dynamic pressure changes can have a dramatic effect on the particle precipitation in the high-latitude ionosphere. It has also been noted that the preexisting interplanetary magnetic field (IMF) orientation can significantly affect the resulting changes in the size, location, and intensity of the auroral oval. Here we focus on the effect of pressure pulses on the size of the auroral oval. We use particle precipitation data from up to four Defense Meteorological Satellite Program (DMSP) spacecraft and simultaneous POLAR Ultra-Violet Imager (UVI) images to examine three events of solar wind pressure fronts impacting the magnetosphere under two IMF orientations, IMF strongly southward and IMF $B_{z}$ nearly zero before the pressure jump. We show that the amount of change in the oval and polar cap sizes and the local time extent of the change depends strongly on IMF conditions prior to the pressure enhancement. Under steady southward IMF, a remarkable poleward widening of the oval at all magnetic local times and shrinking of the polar cap are observed after the increase in solar wind pressure. When the IMF $B_{z}$ is nearly zero before the pressure pulse, a poleward widening of the oval is observed mostly on the nightside while the dayside remains unchanged. We interpret these differences in terms of enhanced magnetospheric reconnection and convection induced by the pressure change. When the IMF is southward for a long time before the pressure jump, open magnetic flux is accumulated in the tail and strong convection exists in the magnetosphere. The compression results in a great enhancement of reconnection across the tail which, coupled with an increase of magnetospheric convection, leads to a dramatic poleward expansion of the oval at all MLTs (dayside and nightside). For near-zero IMF $B_{z}$ before the pulse the open flux in the tail, available for closing through reconnection, is smaller. This, in combination with the weaker magnetospheric convection, leads to a more limited poleward expansion of the oval, mostly on the nightside.
\end{abstract}

Key words. Magnetospheric physics (solar windmagnetosphere interactions; magnetospheric configuration and dynamics; auroral phenomena)

Correspondence to: A. Boudouridis

(thanasis@atmos.ucla.edu)

\section{Introduction}

The solar wind and the accompanying interplanetary magnetic field (IMF) are the main drivers of the dynamics of the terrestrial magnetosphere. Solar wind dynamic pressure enhancements with sufficient duration to engulf the entire magnetosphere have been shown to induce global responses in short time scales to both ionospheric currents and auroral precipitation (Craven et al., 1986; Shue and Kamide, 1998; Zhou and Tsurutani, 1999; Lyons et al., 2000; Zesta et al., 2000; Chua et al., 2001). Prior work has also shown that the magnetosphere responds very differently to long-duration solar wind pressure changes under different IMF conditions. Elphinstone et al. (1991) studied the auroral signatures during two pressure pulses that occurred on 19 October 1986 (IMF data discussed by Winglee and Menietti (1998)). A short-lived pressure pulse under northward IMF had only a limited and localized effect on the aurora. A pressure front under southward IMF produced a global and almost immediate response of the aurora at all local times. Craven et al. (1986) reported similar results. Specifically, the response to a shock front that hit the magnetosphere under southward IMF conditions was a global intensification of the aurora, occurring nearly simultaneously at all MLTs. For a shock that hit under northward IMF conditions there was still a global intensification of the aurora at all local times, but it was not as strong as in the southward IMF case. Zesta et al. (2000) found that a pressure enhancement under strong southward IMF triggers an almost instantaneous and global enhancement of auroral intensity simultaneously at all local times, a significant widening of the auroral oval, and a closing of the polar cap. Chua et al. (2001) also found a simultaneous brightening over broad areas of the dayside and nightside aurora in response to a pressure pulse that occurred with a northward turning of the IMF. In some cases, the auroral disturbance is clearly observed first at the dayside and then it expands to the nightside (Zhou and Tsurutani, 1999; Boudouridis et al., 2003).

In a recent study, Boudouridis et al. (2003) looked at the effect of solar wind pressure enhancements to the auroral intensity, size, and location for three cases with different IMF conditions. They used direct measurements of precipitating 
particle fluxes by up to four Defense Meteorological Satellite Program (DMSP) spacecraft and simultaneous POLAR Ultra-Violet Imager (UVI) images to investigate the dependence of the response of auroral precipitation to an incoming solar wind pressure front on the IMF configuration before and after the front. They concluded that a steady southward IMF throughout the pressure change favors a global intensification of auroral precipitation and ultra-violet (UV) emissions, as well as a dramatic (up to $10^{\circ}$ ) poleward expansion of the oval at all magnetic local times (MLTs). In the case where the IMF $B_{z}$ was nearly zero before the pulse, an increase in auroral precipitation was observed all around the oval but now the poleward motion of the high-latitude oval boundary was seen to a lesser degree and only on the nightside, with no apparent motion on the dayside.

In light of these results Boudouridis et al. (2003) raised a fundamental question: "Why does the auroral oval expand poleward after an enhancement of the solar wind dynamic pressure, and why is this response different for different preexisting IMF configurations?" Boudouridis et al. (2003) suggested that the sudden poleward expansion of the oval away from the noon MLT sector after the arrival of a solar wind pressure front was associated with enhanced tail reconnection triggered by the compression of the magnetosphere. Open field lines close at a higher rate as the open-closed field line boundary (OCB) and thus, the poleward boundary of the auroral oval moves toward higher latitudes. The observations show that the shrinking of the polar cap is more extensive and over a broader MLT range in the case of southward IMF conditions prior to the pressure front impact. Therefore, the strength of the enhanced tail reconnection and its local time variation are dependent on the previous state of the magnetosphere (preconditioning), which, in turn, depends on the preexisting IMF structure. We believe that the surprising result of a poleward motion in the noon sector must also be related to increased reconnection, but how the reconnection process can result in more closed field lines on the dayside is unclear.

In the following sections we discuss observations of the shrinking polar cap (as seen by DMSP particle detectors and POLAR UVI images) and examine how nightside reconnection leads to different results under two different IMF configurations: a) IMF steady southward throughout the pressure pulse event and b) IMF $B_{z}$ nearly zero before the pressure increase. We are able to show that even though the sharp large-scale compression causes enhancement of magnetotail reconnection under both IMF configurations, higher available open flux near the nightside separatrix and much higher convection under southward IMF $B_{z}$ (i.e. preconditioning) are associated with the more spectacular and global changes in the auroral oval under those IMF conditions (both dayside and nightside) compared with the IMF $B_{z} \approx 0$ case (nightside only). In Sect. 2 we describe the effects of a pressure front on the auroral oval and polar cap sizes. In Sect. 3 we discuss the role of magnetospheric reconnection in the different responses observed under different IMF conditions. Finally, in Sect. 4 we summarize our conclusions.

\section{Effects of pressure fronts on aurora}

We examine three case studies occuring under the above IMF conditions. The first one on 10 January 1997 is a pressure pulse event under steady southward IMF. Its auroral response to the pressure pulse has been studied by many authors in the past (e.g. Lyons, 2000; Lyons et al., 2000; Zesta et al., 2000; Boudouridis et al., 2003). Here we give a summary of the previous results on this event which we then compare with the new results for the IMF $B_{z} \approx 0$ case presented in Sect. 2.2. The other two events, on 6 January and 30 April 1998, take place under near-zero IMF $B_{z}$ conditions prior to the pressure front impact.

\section{1 $\operatorname{IMF} B_{z}<0$}

During the widely studied storm of 10 January 1997, a significant and abrupt pressure increase was recorded by the WIND spacecraft at $\sim 10: 30 \mathrm{UT}$, lasted $\sim 22 \mathrm{~min}$ and reached the Earth at $\sim 10: 50$ UT. Its duration, combined with a solar wind velocity of $450 \mathrm{~km} / \mathrm{sec}$, yields a radial size of $\sim 93 R_{E}$ for the high-pressure region in the solar wind, the pressure in it being three times higher than that recorded before and after. This is large enough to engulf and compress the entire magnetosphere. During this event the IMF $B_{z}$ remained strongly negative at all times, following a long period (from 05:00 UT onwards) of strongly southward IMF (Lyons et al., 2000). Therefore, it is an ideal event for studying the effect of a solar wind dynamic pressure enhancement on the size of the polar cap under southward IMF conditions.

Lyons et al. (2000) and Zesta et al. (2000) looked at the effect of the above pressure enhancement on the auroral emissions measured by meridional scanning photometers (MSPs) from the CANOPUS stations at Rankin Inlet and Gillam. They found that the poleward boundary of the $6300 \AA$ and $5577 \AA$ emissions tracks the solar wind density and pressure, moving poleward in response to pressure enhancements, while the emission levels are enhanced during the high pressure interval. Zesta et al. (2000) and Lyons (2000) presented POLAR UVI measurements of the Northern Hemisphere nightside polar region during the period of the above pressure pulse. They showed a significant increase in auroral emissions at around 10:50 UT, accompanied by a poleward motion of the high-latitude auroral boundary (up to $10^{\circ}$ at some MLTs) and a widening of the auroral oval at all visible MLTs. The UV emissions dropped to their prepulse levels at around 11:20 UT, coincident with the solar wind pressure drop. Variations in the auroral emissions were, therefore, directly driven by the solar wind dynamic pressure changes.

Boudouridis et al. (2003) studied the same event, this time using directly measured DMSP precipitating particle fluxes from two spacecraft, F12 and F13. They looked for changes in the size and intensity of the auroral oval caused by the incoming pressure pulse. Figure 1 shows the auroral boundaries before (green) and after (red) the increase in solar wind pressure at 10:50 UT. The individual boundary 


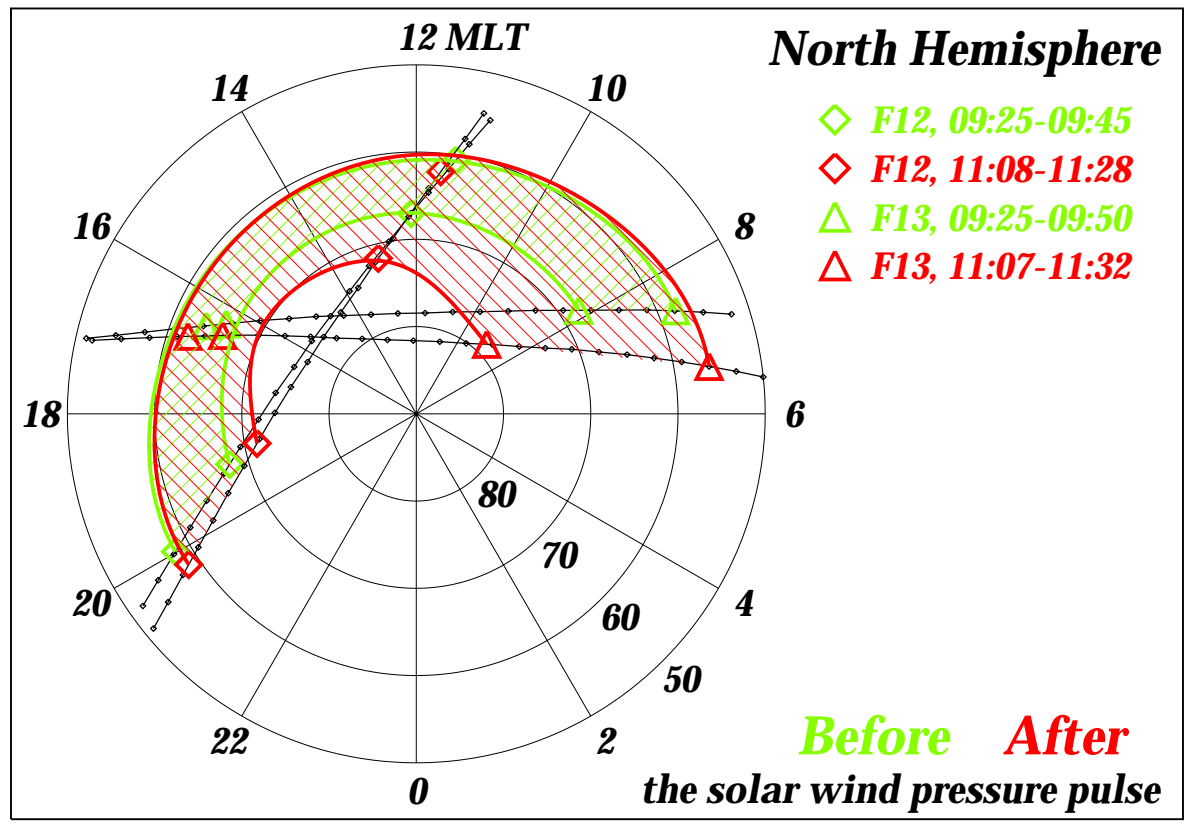

Fig. 1. Auroral boundaries for 10 January 1997, based on the individual boundary identifications from the DMSP spectrograms. The satellite orbits are shown as black lines. The tick marks on them are one minute apart. The big diamonds and triangles represent the actual boundary determinations for F12 and F13, respectively. The green and red curves are the inferred oval boundaries for before and after the arrival of the solar wind pressure front (Boudouridis et al., 2003). A closing of the polar cap is observed at all MLTs.

determinations were based mainly on $2 \mathrm{keV}$ electron differential energy fluxes, as described in Boudouridis et al. (2003), and are marked by the big diamonds and triangles for F12 and F13, respectively. The green and red curves are the oval boundaries before and after the arrival of the solar wind pressure front, derived by a simple spline fit on the actual boundary crossings which was then smoothed to produce a more continuous boundary. The high-latitude boundary moves clearly poleward in the region of $\sim 8-20$ MLT after the pressure jump. This motion is a few degrees in the dusk region and as much as $10^{\circ}$ in the dawn region. Furthermore, Boudouridis et al. (2003) showed that the total energy input into the ionosphere due to particle precipitation greatly increased after the pressure pulse impact, covering a substantially wider auroral oval, with fluxes of the order of $10^{11}-10^{12} \mathrm{eV} /\left(\mathrm{cm}^{2}\right.$ ster $\left.\mathrm{sec}\right)$.

The simultaneous widening of the oval and shrinkage of the polar cap on both the dayside (DMSP data, (Boudouridis et al., 2003)) and the nightside (UV data, (Lyons, 2000; Zesta et al., 2000)) signifies a global response of the magnetosphere-ionosphere system to the incoming pressure pulse. This response is energetically very important and, as we show later on, closely associated with the preexisting state of the magnetosphere.

\subsection{IMF $B_{z} \approx 0$ before the pressure change}

Boudouridis et al. (2003) also studied an event on 2 October 1998, where the IMF $B_{z}$ was close to zero before the pressure increase and substantially fluctuated after. Their main conclusion was that the auroral precipitation response in this case was not as strong as in the southward-IMF 10 January 1997 case. The dayside oval did not show a significant change in its size but the auroral UV emissions did increase after the pressure shock, with the emissions' intensification exhibiting a small noon-to-midnight propagation, especially in the Northern Hemisphere duskside. On the nightside, there was a substantial poleward widening of the oval and increased particle precipitation, seen in both the DMSP and Polar UV observations. However, the closing of the polar cap on the nightside was again less dramatic compared with the southward-IMF 10 January 1997 event.

The nightside intensification and poleward motion resembles the effects of a substorm. However, the auroral observations as well as geosynchronous energetic particle data (e.g. Fig. 14 of Boudouridis et al. (2003)), clearly showed that the general response is not due to substorms. In particular, the effects of the pressure fronts occur over a wide range of MLTs as opposed to the more localized (premidnight) substorm features. Also, ionospheric currents show an enhancement in the large-scale DP2 current, and do not show the nightside enhancement of the westward electrojet that is associated with the substorm current wedge (Zesta et al., 2000).

In order to better understand the behavior of the auroral oval in this near-zero preceding IMF $B_{z}$ scenario and test the generality of the results obtained for the 2 October 1998 event, we examine two more events of this kind. 


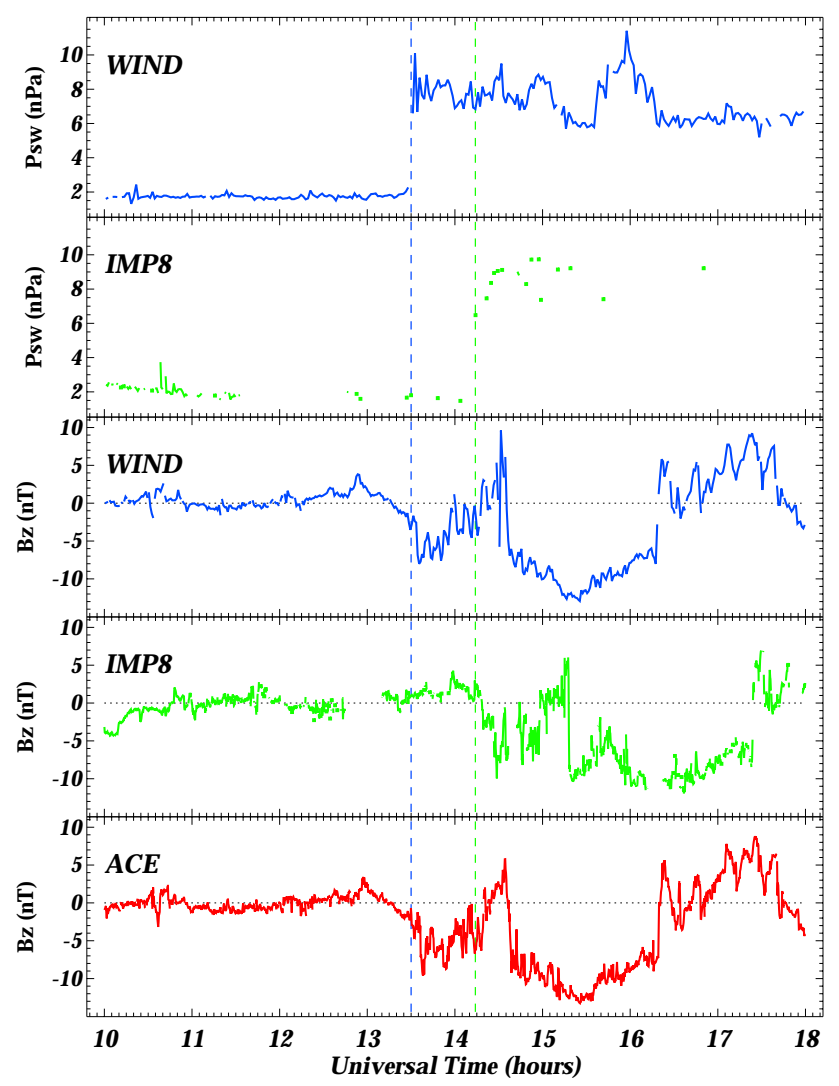

Fig. 2. Solar wind conditions for 6 January 1998. The top two panels show the solar wind dynamic pressure observed by WIND and IMP8, while the three bottom panels show the IMF $B_{z}$ component in GSE coordinates measured by WIND, IMP8, and ACE. The three spacecraft were located at $X_{\mathrm{GSE}} \sim 227 R_{E}, X_{\mathrm{GSE}} \sim 30 R_{E}$, and $X_{\mathrm{GSE}} \sim 220 R_{E}$, for WIND, IMP8, and ACE, respectively. The vertical lines mark the times when WIND (blue) and IMP8 (green) encountered the pressure front. The pressure front impacted the magnetosphere at $\sim 14: 25 \mathrm{UT}$.

\subsubsection{Event 1: 6 January 1998}

Figure 2 shows solar wind pressure and IMF $B_{z}$ measurements for the pressure increase on 6 January 1998 at $\sim 14: 25$ UT. The top two panels show the solar wind dynamic pressure observed by WIND and IMP8, while the three bottom panels show the IMF $B_{z}$ component in GSE coordinates measured by WIND, IMP8, and ACE. The solar wind pressure front was first detected by WIND at $\sim 13: 30$ UT. The dynamic pressure exhibited a step change from $2 \mathrm{nPa}$ to $\sim 8 \mathrm{nPa}$ and then remained high at $\sim 8 \mathrm{nPa}$ for many hours. The impact at the magnetosphere was estimated to be at $\sim 14: 20$ 14:25 UT consistent with the IMP8 detection of the pressure front at $\sim 14: 15 \mathrm{UT}$ at $X_{\mathrm{GSE}} \sim 30 R_{E}$. The IMF $B_{z}$ preceding this pressure front was almost zero for many hours before, and at impact it turned negative and remained mostly negative until $\sim 17: 25$ UT. There was only a short-lived northward turning at $\sim 15: 20 \mathrm{UT}$.

The polar maps of the DMSP boundary identifications (based on three DMSP spacecraft) for this event are shown in
Fig. 3. The format of this plot is the same as in Fig. 1, with the Northern Hemisphere passes shown on the top and Southern Hemisphere passes shown on the bottom panel. Figure 3 indicates that the size and location of the dayside auroral oval remained unchanged for both hemispheres from before (green) to after (red) the solar wind pressure change. Notice that the poleward boundary of the oval before the pressure enhancement was already at high latitude $\left(\sim 78^{\circ}\right)$ as compared with the same boundary for the 10 January 1997 case (at $\sim 68^{\circ}$ ).

The nightside sector is better seen in the POLAR UVI data shown in Fig. 4 for the Northern Hemisphere. Nine images of derived electron energy flux are shown, one every $\sim 6 \mathrm{~min}$, from $\sim 14: 01$ UT (top left) until $\sim 14: 50$ UT (bottom right). The three circles in each image correspond to magnetic latitudes of $60^{\circ}, 70^{\circ}$, and $80^{\circ}$. An intensification is clearly observed at all available MLTs (within the field of view of POLAR at that time) at $\sim 14: 25$ UT (5th image), with intensity increasing from $\sim 3$ to $\sim 7 \mathrm{~mW} / \mathrm{m}^{2}$ in the postmidnight region. A poleward expansion of the oval begins at the same time on the nightside, reaching $\sim 75^{\circ}$ of latitude at $\sim 14: 44$ UT. It is important to point out here that the closing of the polar cap on the nightside occurs at the same time as the IMF $B_{z}$ turns negative, which would normally open the polar cap. However, for this event the effects of the pressure enhancement (i.e. closing of the polar cap) counteract the effects of the IMF southward turning.

\subsubsection{Event 2: 30 April 1998}

For the second event we concentrate on the period 04:0016:00 UT on 30 April 1998. The IMF $B_{z}$ and solar wind dynamic pressure data for this period are shown in Fig. 5. The top three panels show the solar wind dynamic pressure observed by WIND, IMP8, and ACE, while the three bottom panels show the IMF $B_{z}$ component in GSE coordinates measured by the same spacecraft. All three spacecraft record a remarkably similar behavior of both the dynamic pressure and the IMF $B_{z}$ component. The pressure, being quite low for many hours prior to the front $(\sim 2-3 \mathrm{nPa})$, jumps to a $12-$ $14 \mathrm{nPa}$ value and remains at this high level for about $4 \mathrm{~h}$. The IMF $B_{z}$, on the other hand, stays more or less stable at nearzero values throughout the pressure change, exhibiting only a slightly higher degree of variability under the high pressure. The arrival time of the front at the nose of the magnetosphere is estimated at $\sim 09: 25 \mathrm{UT}$, in agreement with the IMP8 crossing at $\sim 09: 20$ UT. This event is ideally suited for examining the effects of solar wind dynamic pressure enhancements under IMF $B_{z} \approx 0$ conditions throughout the pressure change.

The oval location based on the DMSP particle characteristics is shown in Fig. 6 in the same format as in Fig. 3. In the Northern Hemisphere (top), no satellite crosses the poleward boundary of the dayside oval (green shading) before the pressure increase. The highest latitude reached (by F11) is $\sim 76^{\circ}$, and thus, the boundary lies poleward of that latitude. After the front impact, the poleward boundary is detected by F11 

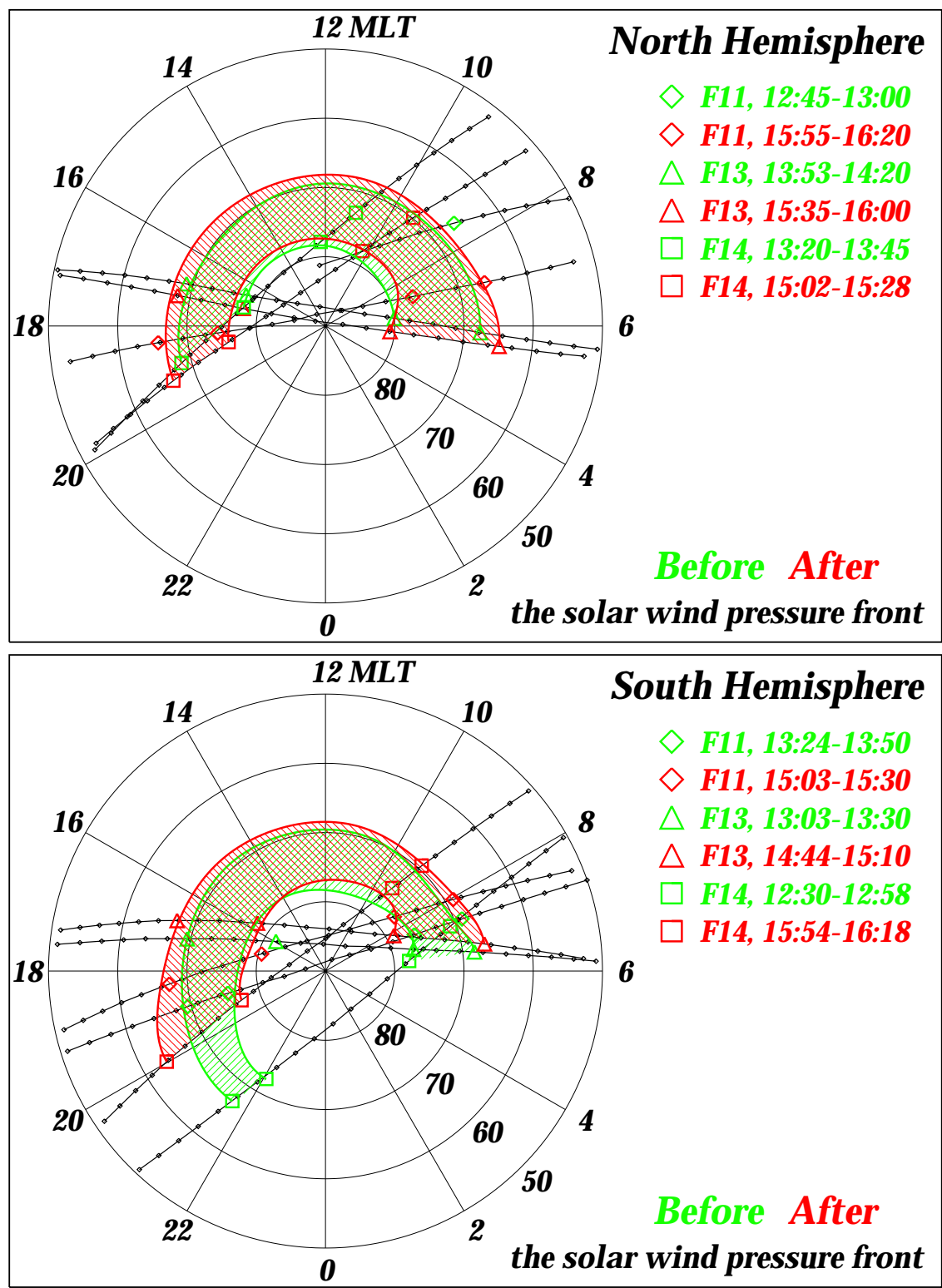

Fig. 3. The same as in Fig. 1 for 6 January 1998, North Hemisphere on the top and South Hemispere on the bottom. The various symbols denoting the boundary crossings by different spacecraft are explained at the legend on the right. No poleward motion of the dayside oval is observed.

at around the same latitude, and by $\mathrm{F} 13$ at $80^{\circ}$. Therefore, it is uncertain if any poleward motion took place at the dayside due to the pressure enhancement. However, considering again the very high starting point of the poleward boundary ( $>76^{\circ}$ before the pulse), there was most likely no significant closing of the polar cap at the dayside. In the Southern Hemisphere (bottom panel) the situation is very different. The nightside oval responds to the change in solar wind pressure by expanding poleward by about $5^{\circ}$, as observed by the F11 and F13 spacecraft which crossed the high-latitude oval boundary both before and after the high pressure impact. Since the oval's equatorward boundary remains more or less stationary, the result is a significant increase in the oval width.
There are no UV images for this event, but the change in the southern nightside oval size, together with an increase in auroral particle precipitation, are clearly demonstrated in the electron integral flux plots of Fig. 7. The two panels on the left depict, as a color-coded trace, the electron integral energy flux measured along the DMSP orbits before (top) and after (bottom) the increase in solar wind dynamic pressure. The panels on the right show simple two-dimensional interpolations of the trace fluxes. Both display formats show a much wider oval with intensity about an order of magnitude higher in most locations in the "after" panels (bottom) as compared with the "before" panels (top). 

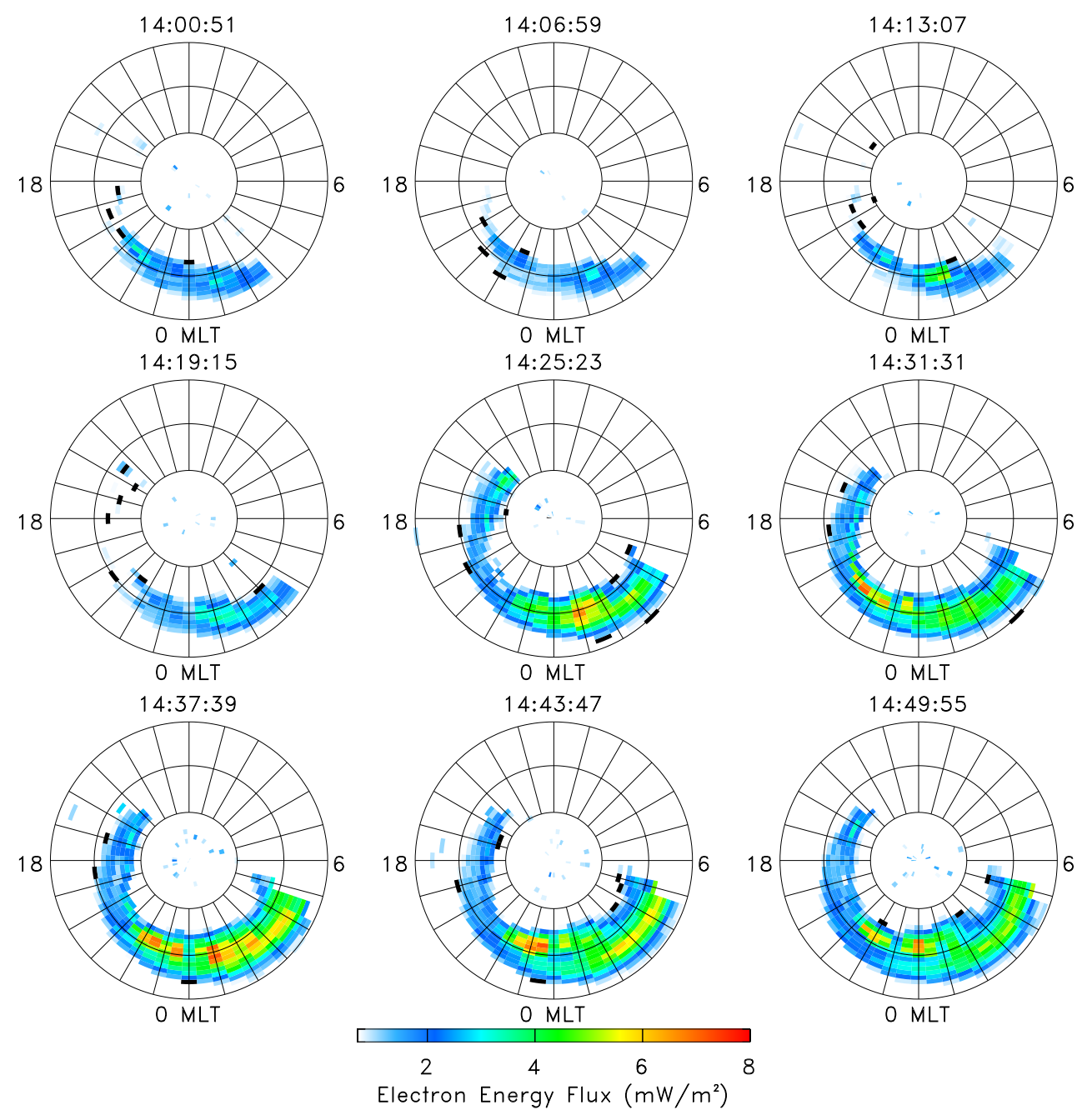

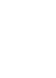

Fig. 4. POLAR UVI measurements for 6 January 1998. Nine images of derived electron energy flux are shown, one every $\sim 6$ min, starting at $\sim$ 14:01 UT (top left) until $\sim 14: 50$ UT (bottom right). An intensification is observed at all available MLTs at $\sim 14: 25$ UT (5th image), and a poleward expansion of the oval is observed on the nightside. The absence of dayside UV emissions in this plot is due to the limited field of view of the POLAR spacecraft. An enhancement of auroral precipitation was indeed observed in the dayside oval by the DMSP spacecraft.

\section{The role of magnetospheric reconnection}

As mentioned in the Introduction, an important question arises now (Boudouridis et al., 2003): "What causes the auroral oval to expand poleward and the polar cap size to diminish in response to the compression of the magnetosphere?" Boudouridis et al. (2003) briefly discussed the various possibilities and concluded that the large and rapid shrinking of the polar cap that occurs in response to dynamic pressure enhancements corresponds to a large and rapid decrease in the amount of open polar cap magnetic flux. Such a conversion of open to closed flux must be a manifestation of reconnection. But why is the response different under different preexisting IMF conditions? What leads to the tremendous poleward widening of the oval at all MLTs in the southward IMF case as opposed to the more limited nightside motion in the near-zero $B_{z}$ case?
We argue that most of the polar cap shrinkage is associated with increased nightside reconnection, and that the strength and MLT extent of this enhanced magnetotail reconnection depends on the previous state of the magnetosphere. A summary of our observations and current understanding of how this works under different preexisting IMF orientations to produce the observed polar cap shrinkage, is given in the schematic representation of the polar region in Fig. 8. The top panel describes the auroral response when IMF $B_{z}<0$ and the bottom panel describes the situation when IMF $B_{z} \approx 0$ before the front impact. The blue solid lines mark the location of the poleward boundary of the oval before the pressure increase, and the red ones mark the position of the same boundary as observed after the pressure jump. The solid green line denotes the poleward boundary of the region where field lines that map to the tail, close as a result of the pressure enhancement. This simple drawing does not include the effects of an IMF $B_{y}$ component that would distort the regions drawn along the dawn-dusk direction. 


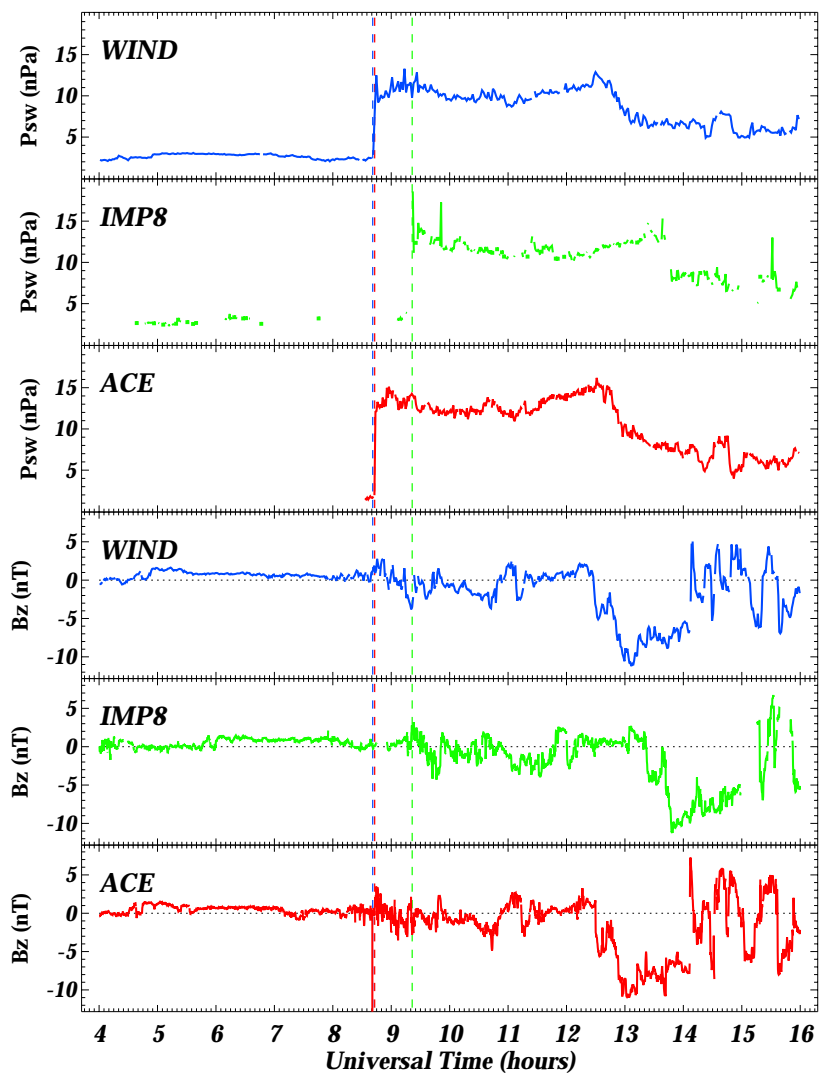

Fig. 5. Solar wind conditions for 30 April 1998. The top three panels show the solar wind dynamic pressure observed by WIND, IMP8, and ACE, while the three bottom panels show the IMF $B_{z}$ component in GSE coordinates measured by the same spacecraft. WIND, IMP8, and ACE were located at $X_{\mathrm{GSE}} \sim 217 R_{E}$, $X_{\mathrm{GSE}} \sim 27 R_{E}$, and $X_{\mathrm{GSE}} \sim 229 R_{E}$, respectively. The vertical lines mark the detection times of the front by the three spacecraft. The high pressure front impacted the magnetosphere at $\sim 09: 25$ UT.

In the first case (top), the strong negative IMF $B_{z}$ (present for a long period before the pulse) has acted to open the polar cap wide (blue line), resulting in a large region of open field lines mapping back into the tail (green hatched area). When the pressure front impacts the magnetosphere, enhanced reconnection in the tail closes these open field lines as the OCB moves poleward. This area occupies not only the nightside portion of the polar cap, but also extends along the dawn/dusk flanks. This produces much of the global response seen in cases like 10 January 1997. However, this cannot account for the dayside polar cap closing near noon (red hatched area) observed in this event.

In the $B_{z} \approx 0$ case (bottom panel) the polar cap is already considerably closed before the arrival of the pressure front (the dashed blue line is the location of the poleward oval boundary for IMF $B_{z}<0$, shown for comparison). The tail field lines below the solid blue line have already been closed due to the prolonged near-zero IMF $B_{z}$. Therefore, when the pressure front hits the magnetosphere, enhanced tail reconnection closes only a smaller portion of open tail field lines (green hatched area in bottom panel) compared with the southward IMF case. This results in a diminished observable response of the size and location of the auroral oval, limited mostly to the nightside. The dayside oval does not show any size or location variation, as was seen for the 6 January and 30 April 1998 events.

Additional information on the different responses for the two IMF cases can be obtained by using the Tsyganenko96 (hereinafter referred to as T96) geomagnetic field model (Tsyganenko, 1995, 1996). Despite the limitations of this model in determining the separatrix between the open and closed field lines in the tail, qualitative results can be derived showing the difference in the magnetospheric configuration before a pressure front impact for the two IMF conditions, that leads through enhanced magnetotail reconnection to the different ionospheric responses observed by the DMSP spacecraft.

Figure 9 shows results of T96 field line tracing for the conditions present right before the solar wind pressure enhancement $\left(P_{s w}=2 \mathrm{nPa}\right)$. It depicts how the field magnitude and location in the magnetotail region defined by $X_{\mathrm{GSM}}=-20 R_{E}$, $-30 R_{E}<Y_{\mathrm{GSM}}<30 R_{E}, 0 R_{E}<Z_{\mathrm{GSM}}<20 R_{E}$, map down to the source of the field lines in the high-latitude ionosphere for the two IMF conditions preceding the pressure front arrival, strongly southward IMF (top) and near-zero IMF $B_{z}$ (bottom). The dipole tilt and the IMF $B_{y}$ component were assumed to be zero in this simulation, to guarantee northsouth and dawn-dusk symmetry of the results. The colorcoded images represent the total field magnitude in the tail, expressed in $n T$, according to the color scale at the right. The solid black line gives the approximate location of the separatrix. This was determined by tracing the field lines to the last point, near $100 R_{E}$ downtail, and checking to see if they crossed the equatorial plane $\left(Z_{G S M}=0\right)$. All field lines that reached $100 R_{E}$ downtail without crossing the equatorial plane were defined as open. Obviously, this criterion is not entirely accurate as some field lines might close further downtail, moving the actual position of the separatrix to higher $Z_{G S M}$.

However, the uncertainty in the exact location of the separatrix does not affect our qualitative conclusions here. The difference in the field strength in the tail for the two cases is what really matters. In the top panel the field is accumulated in the tail as a result of the negative IMF $B_{z}$. This region of high tail field strength maps to the polar cap area in which the rapid and widespread poleward widening of the oval is observed by the DMSP satellites. Our observations show that, under these conditions, a compression of the magnetosphere is related to reconnection along the separatrix region all across the tail. The enhanced tail magnetic field could be the reason why tail reconnection is more efficient in closing open magnetic flux over a wide range of MLTs for the southward IMF case. The same polar cap area in the near-zero $B_{z}$ case maps to a tail region with $30 \%$ lower field magnitude. The total magnetic flux in the near-separatrix region, for example, in a strip $3 R_{E}$ above the separatrix, is $24 \%$ lower in this case. Enhanced reconnection taking place in the tail for 

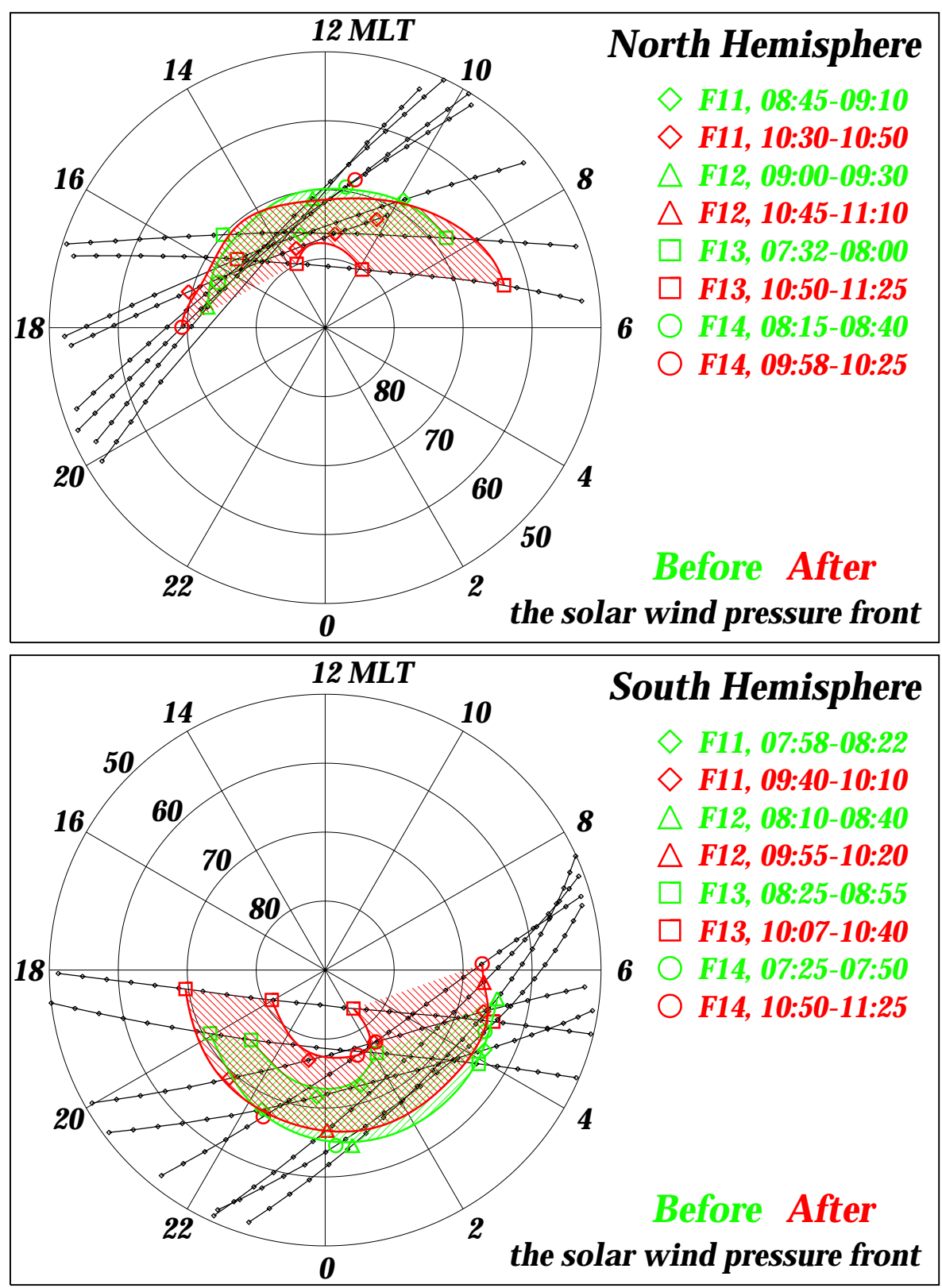

Fig. 6. The same as in Fig. 3 for 30 April 1998, North Hemisphere on the top and South Hemispere on the bottom. The various symbols denoting the boundary crossings by different spacecraft are explained at the legend on the right. A significant poleward expansion of the nightside South Hemispere oval is observed.

this configuration closes the polar cap on the nightside where the tail field strength is still high, but not on the flanks where the tail field strength is weaker.

Enhanced magnetotail reconnection can explain the polar cap shrinkage observed in the IMF $B_{z} \approx 0$ cases. But it still cannot fully explain the southward IMF case. The closing of the polar cap in the 8-16 MLT range suggests the contribution of another process that "closes" field lines on the dayside. There is a possible connection to the enhanced magnetotail reconnection which is opposite from what is normally expected for IMF $B_{z}<0$. If the increased reconnection in the tail is cou- pled with enhanced magnetospheric convection induced by the pulse, then it is possible to transport the newly-closed tail/flank field lines to the dayside in much shorter time scales than usual.

Evidence of enhanced ionospheric convection can be found during the 10 January 1997 event in the DMSP electric field data and the associated ionospheric flows. Figure 10 shows data from two DMSP F13 passes, before (left) and after (right) the pressure pulse event. Each plot shows (from top to bottom) ionospheric horizontal velocity, electron integral energy flux, electron differential energy flux, ion integral energy flux, and ion differential energy flux. The spacecraft 

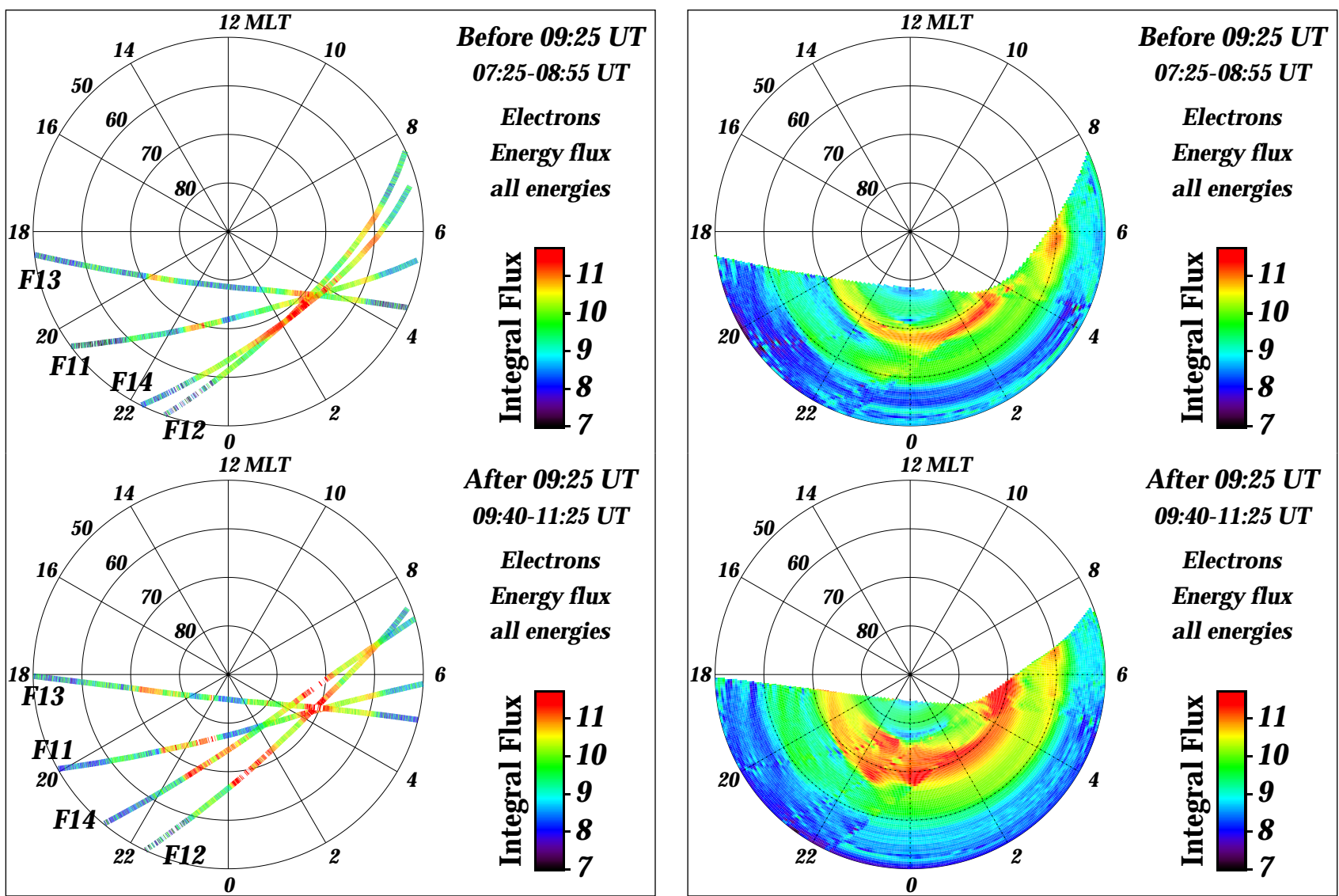

Fig. 7. DMSP electron integral energy fluxes for the Southern Hemisphere on 30 April 1998. The two panels on the left are the actual measurements before (top) and after (bottom) the solar wind pressure front. The panels on the right are two-dimensional interpolations of the trace fluxes on the left. An intensification and poleward expansion of the aurora are clearly observed.

orbit is shown in the inset to the right of the electron integral energy flux. The auroral oval crossings for these passes are marked by the hatched boxes above the electron integral energy flux curves, and correspond to the regions marked by the triangles along the F13 orbits in Fig. 1. The ionospheric velocity is measured in the direction perpendicular to the spacecraft orbit, which in this case is almost along the noon-midnight direction. Positive velocities are sunward and negative velocities are antisunward. The "after" pass shows dramatically enhanced flows all along the satellite orbit. Inside the dawn oval, which expanded poleward by $\sim 10^{\circ}$, the ionospheric velocity increased from $\sim 600 \mathrm{~m} / \mathrm{s}$ to $\sim 1500-2000 \mathrm{~m} / \mathrm{s}$. This high convection velocity can transport a newly-closed field line along the $70^{\circ}$ latitude circle from the flanks ( $\sim 5 \mathrm{MLT})$ to noon in about $30 \mathrm{~min}$, and in even shorter times for higher latitudes. F13 crossed the expanded poleward oval boundary after the pressure front at 11:22 UT, $\sim 32 \mathrm{~min}$ after the front impact at $\sim 10: 50 \mathrm{UT}$.

Therefore it is possible that increased convection in the magnetosphere can move the newly-reconnected field lines piling up at the nightside and flanks to the dayside after the pressure front impact. When this convection is as high as in the southward IMF case, a large amount of closed flux can be transported rapidly to the dayside, producing the closing of the polar cap observed there for this case. Enhanced ionospheric convection has also been observed for the IMF $B_{z} \approx 0$ cases, but of considerably smaller magnitude which renders it unable to transfer newly-closed flux from the tail to the dayside in short time scales.

Finally, we would like to comment briefly on how the IMF orientation after the pressure change affects the above auroral response. Boudouridis et al. (2003) showed an event on 18 February 1999, where the IMF was strongly negative before and it turned strongly positive after the increase in solar wind pressure. According to the above interpretation for the IMF $B_{z}<0$ case, we again expect to see closing of the polar cap. And this was actually observed on the dayside. The difference now is that the northward turning of the IMF can also result in the closing of the polar cap and the two effects are not easily distinguishable. The poleward widening of the oval in this case was detected by the DMSP F12 spacecraft 48 min after the simultaneous front impact and IMF northward turning. This time is long enough for the reconfiguration of the polar cap convection due to changes in IMF orientation, $\sim 12-24 \mathrm{~min}$ (Ridley et al., 1997), and the closing of the polar cap due to the pressure pulse, $\sim 10-15 \mathrm{~min}$ 


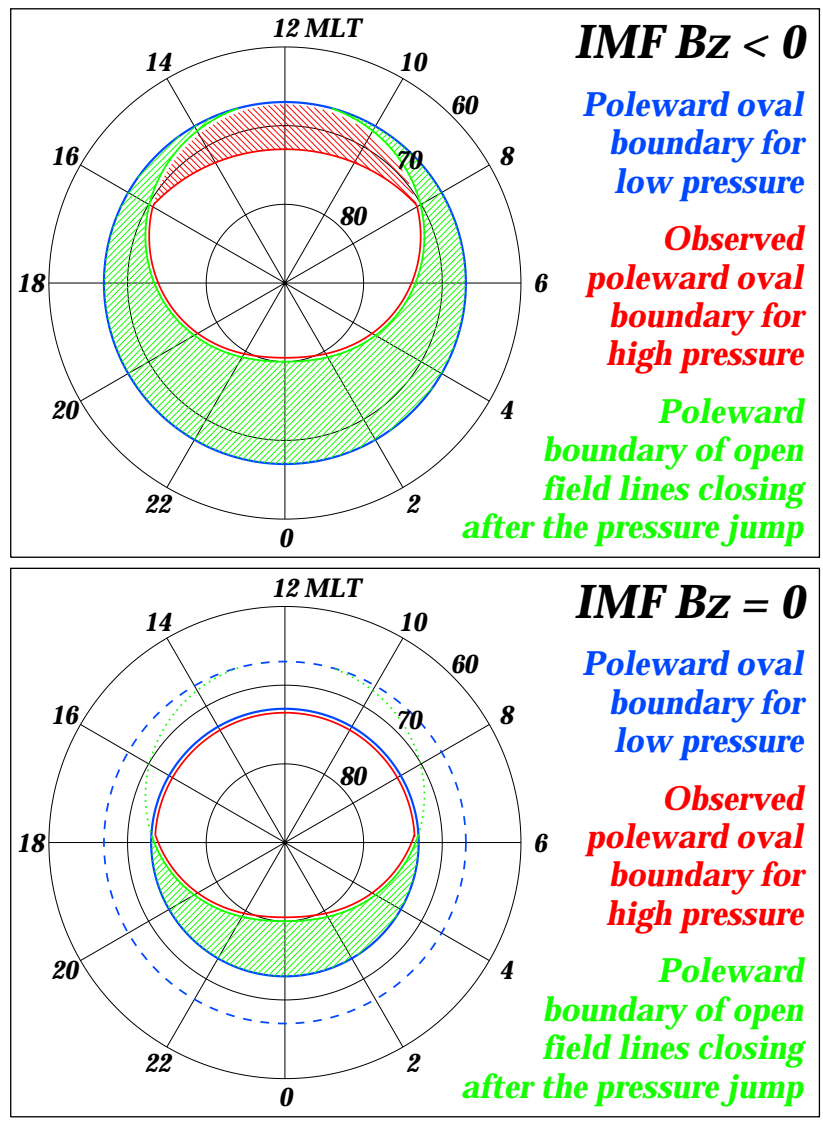

Fig. 8. A diagram illustrating the role of enhanced magnetotail reconnection in closing the polar cap after the arrival of the pressure front, for IMF $B_{z}<0$ (top) and IMF $B_{z} \approx 0$ (bottom). The green hatched area in both panels represents the area of previously open tail field lines now closing due to the pressure enhancement. This mechanism, however, cannot account for the dayside polar cap closing when IMF $B_{z}<0$ (red hatched area on the top panel).

(Zesta et al., 2000), to take place. Most likely, both effects contributed to the closing of the polar cap in this case. In addition, a simultaneous substorm developed on the nightside and obscured the effects of the pressure change there. The two events under near-zero preceding IMF $B_{z}$ that we studied here (Sect. 2.2) have a different IMF $B_{z}$ orientation after the pressure jump, one turns southward and the other remains zero, but this does not produce any observable differences in the DMSP or POLAR UVI responses.

\section{Conclusions}

We can summarize our observations as follows. When the IMF is strongly southward before the increase in solar wind dynamic pressure, the oval responds most spectacularly to the impacting front. A poleward expansion of the oval is witnessed at all MLTs, more or less simultaneously, ranging from a few degrees to up to $10^{\circ}$ at some longitudes. This tremendous increase in the oval size and the ensuing shrinking of the polar cap area are accompanied by an enhancement
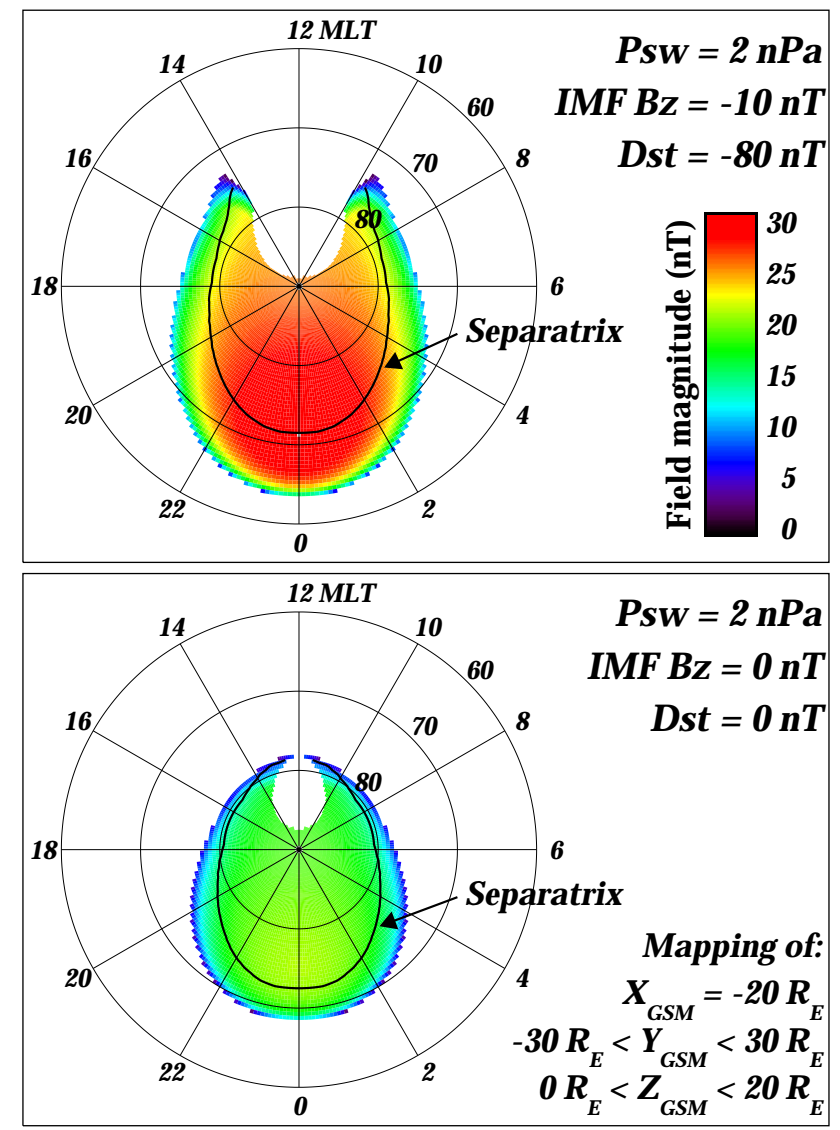

Fig. 9. Results of the Tsyganenko-96 geomagnetic field model for the two IMF cases prior to the pressure enhancement $\left(P_{s w}=2 \mathrm{nPa}\right)$, IMF strongly southward (top), and IMF $B_{z}=0$ (bottom). The colorcoded images represent the mapping to the ionosphere of the total field magnitude in the tail $\left(X_{\mathrm{GSM}}=-20 R_{E}\right)$, expressed in $n T$ according to the color scale at the right. The solid black line gives the approximate location of the separatrix. The field strength in the top panel is $30 \%$ higher than that in the bottom panel, resulting in a more efficient reconnection enhancement after the pressure front impact, that leads to a more widespread shrinking of the polar cap.

of the precipitating particles energy input in the ionosphere all around the oval. This is a global response, characteristically different from the more localized effects of substorms (e.g. Boudouridis et al., 2003). When IMF $B_{z} \approx 0$ before the pressure front impact, the auroral precipitation reacts differently than when IMF $B_{z}<0$. An intensification of the oval occurs again at all MLTs, but in this case a poleward expansion of the oval takes place predominantly on the nightside, whereas the dayside oval seems unchanged in size and location.

These observations strongly support the idea that preconditioning plays an important role in the response of the magnetosphere to the incoming solar wind pressure fronts. Specifically, we associate the closing of the polar cap with an enhancement of magnetotail reconnection resulting from the compression of the magnetosphere by the high-pressure solar wind, because the reduction of open polar cap flux observed 


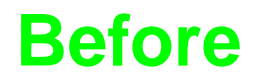

F13 Particle and Field Data, January 10, 1997

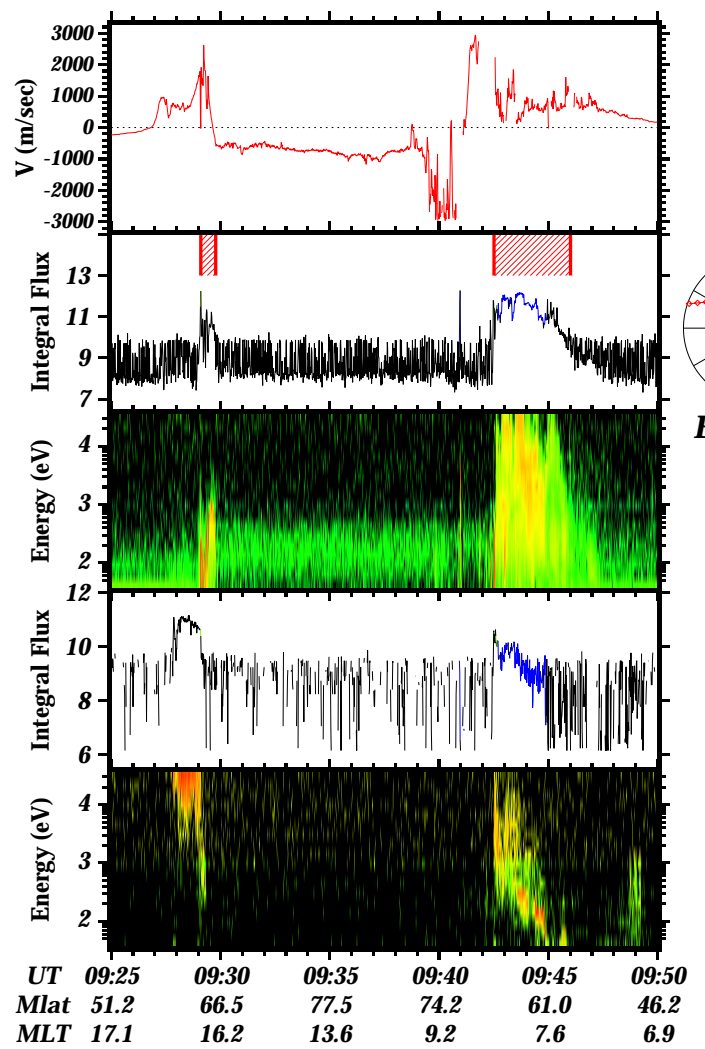

After

F13 Particle and Field Data, January 10, 1997

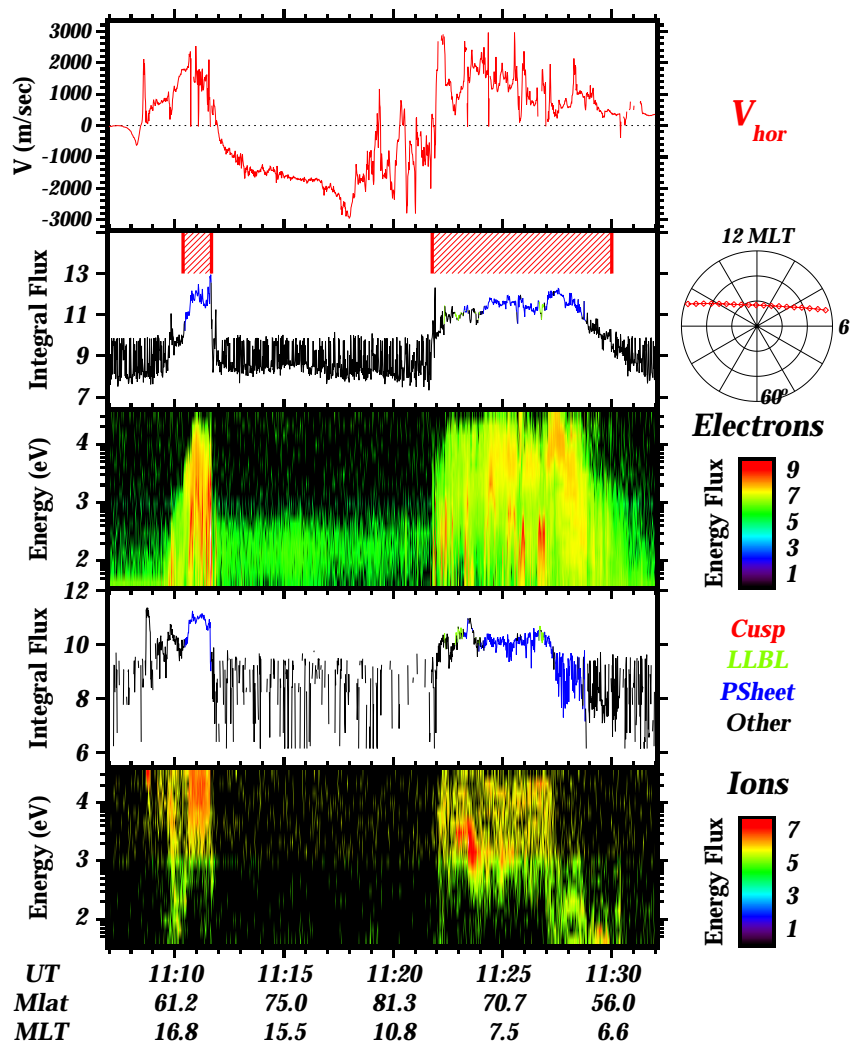

Fig. 10. DMSP F13 particle and electric field data for 10 January 1997, before (left) and after (right) the pressure pulse event. Each plot shows (from top to bottom) horizontal ionospheric velocity, electron integral energy flux, electron differential energy flux, ion integral energy flux, ion differential energy flux. The spacecraft orbit is shown in the inset to the right of the electron integral energy flux. The red hatched boxes above the electron integral flux curves denote the oval locations as determined in Fig. 1. The ionospheric velocity is measured perpendicular to the spacecraft orbit, in this case almost along the day-night direction, sunward when positive and antisunward when negative. Dramatically enhanced flows all along the satellite path are seen in the "after" pass.

requires a significant closing of field lines. Furthermore, we suggest that the magnitude and local time variation of this reconnection enhancement are controlled by the IMF orientation prior to the arrival of the pressure front.

When the IMF is southward for a long time before the pressure jump, the polar cap is wide open, the magnetotail is loaded with energy (open, stretched field lines, closely packed together), and in general, the magnetosphere is very "receptive" to a sudden change in solar wind pressure. The result is a great enhancement of reconnection across the tail, which, coupled with an increase of magnetospheric convection, leads to a dramatic poleward expansion of the oval and shrinking of the polar cap at all MLTs. On the other hand, when IMF $B_{z}$ is almost zero before the pulse, the magnetic flux of open field lines in the tail, available for closing through reconnection, is smaller. This, in combination with the weaker enhancement of ionospheric convection due to the pressure change, results in a more limited poleward expansion of the oval, mostly on the nightside.
In conclusion, we can reliably say that the preexisting IMF structure affects the ability of the magnetosphere to respond to the incoming pressure change. On the other hand, the IMF conditions following the pressure enhancement are important in our ability to clearly observe and isolate this response from other concurrent processes. More events need to be studied, encompassing the full spectrum of IMF orientation before and after the pressure front impact. Also, the above conclusion of enhanced magnetotail reconnection as a response to an increase in solar wind pressure, could be further investigated by looking for signatures of enhanced reconnection and convection, both at low altitudes (e.g. DMSP data) and locally in the tail (e.g. Geotail data). 
Acknowledgements. The authors wish to acknowledge N. Ness at Bartol Research Institute, A. Szabo and R. P. Lepping at NASA GSFC, D. J. McComas at LANL, A. Lazarus at MIT, K. Ogilvie at NASA GSFC, and CDAWeb for the use of IMF and plasma data from the ACE, IMP8, and WIND spacecraft. This work was supported by NASA grant NAG5-12007 and NSF grant OPP-0136139. P. C. Anderson acknowledges support from NSF grant NSF-ATM0000268. Topical Editor T. Pulkkinen thanks K. Nykiri and another referee for their help in evaluating this paper.

\section{References}

Boudouridis, A.: Zesta, E., Lyons, L. R., Anderson, P. C., and Lummerzheim, D.: Effect of solar wind pressure pulses on the size and strength of the auroral oval, J. Geophys. Res., 108(A4), 8012, doi:10.1029/2002JA009373, 2003.

Chua, D., Parks, G., Brittnacher, M., Germany, G., and Spann, J.: Energy characteristics of auroral electron precipitation: A comparison of substorms and pressure pulse related auroral activity, J. Geophys. Res., 106, 5945-5956, 2001.

Craven, J. D., Frank, L. A., Russell, C. T., Smith, E. J., and Lepping, R. P.: Global auroral responses to magnetospheric compressions by shocks in the solar wind: Two case studies, in: Solar WindMagnetosphere Coupling, edited by Kamide, Y., and Slavin, J. A., 367-380, Terra Scientific, Tokyo, 1986.

Elphinstone, R. D., Murphree, J. S., Cogger, L. L., Hearn, D., and Henderson, M. G.: Observations of changes to the auroral distribution prior to substorm onset, in: Magnetospheric Substorms, Geophys. Monogr. Ser., vol. 64, edited by Kan, J., Potemra, T. A., Kokubun, S., and Iijima, T., 257, AGU, Washington, D. C., 1991.
Lyons, L. R.: Geomagnetic disturbances: characteristics of, distinction between types, and relations to interplanetary conditions, J. Atmos. Solar-Terr. Phys., 62, 1087-1114, 2000.

Lyons, L. R., Zesta, E., Samson, J. C., and Reeves, G. D.: Auroral disturbances during the January 10, 1997 magnetic storm, Geophys. Res. Lett., 27, 3237-3240, 2000.

Ridley, A. J., Gang, L., Clauer, C. R., and Papitashvili, V. O.: Ionospheric convection during nonsteady interplanetary magnetic field conditions, J. Geophys. Res., 102, 14,563-14,579, 1997.

Shue, J.-H. and Kamide, Y.: Effects of solar wind density on the westward electrojet, in: SUBSTORMS-4, edited by Kokubun, S. and Kamide, Y., 677-680, 1998.

Tsyganenko, N. A.: Modeling the Earth's magnetospheric magnetic field confined within a realistic magnetopause, J. Geophys. Res., 100, 5599-5612, 1995.

Tsyganenko, N. A.: Effects of the solar wind conditions on the global magnetospheric configuration as deduced from data-based field models, in Proceedings of the ICS-3 Conference on Substorms, Eur. Space Agency Spec. Publ., vol. 389, 181-185, 1996.

Winglee, R. M. and Menietti J. D.: Auroral activity associated with pressure pulses and substorms: A comparison between global fluid modeling and Viking UV imaging, J. Geophys. Res., 103, 9189-9205, 1998.

Zesta, E., Singer, H. J., Lummerzheim, D., Russell, C. T., Lyons, L. R., and Brittnacher, M. J.: The effect of the January 10, 1997, pressure pulse on the magnetosphere-ionosphere current system, in: Magnetospheric Current Systems, Geophys. Monogr. Ser., vol. 118, edited by Ohtani, S., Fujii, R., Hesse, M., and Lysak, R. L., 217-226, AGU, Washington, D. C., 2000.

Zhou, X. and Tsurutani, B. T.: Rapid intensification and propagation of the dayside aurora: Large scale interplanetary pressure pulses (fast shocks), Geophys. Res. Lett., 26, 1097-1100, 1999. 